\title{
Turbulent characteristics of saltation and uncertainty of saltation model parameters
}

\author{
Dongwei Liu ${ }^{1}$, Masahide Ishizuka ${ }^{2}$, Masao Mikami ${ }^{3}$, and Yaping Shao ${ }^{4}$ \\ ${ }^{1}$ School of Ecology and Environment, Inner Mongolia University, Hohhot, China \\ ${ }^{2}$ Faculty of Engineering and Design, Kagawa University, Kagawa, Japan \\ ${ }^{3}$ Office of Climate and Environmental Research Promotion, Japan Meteorological Business Support Center, Tokyo, Japan \\ ${ }^{4}$ Institute for Geophysics and Meteorology, University of Cologne, Cologne, Germany
}

Correspondence: Yaping Shao (yshao@uni-koeln.de)

Received: 22 November 2017 - Discussion started: 19 December 2017

Revised: 28 March 2018 - Accepted: 3 May 2018 - Published: 31 May 2018

\begin{abstract}
It is widely recognised that saltation is a turbulent process, similar to other transport processes in the atmospheric boundary layer. Due to a lack of high-frequency observations, the statistic behaviour of saltation is so far not well understood. In this study, we use the data from the Japan-Australia Dust Experiment (JADE) to investigate the turbulent characteristics of saltation by analysing the probability density function, energy spectrum and intermittency of saltation fluxes. Threshold friction velocity, $u_{* t}$, and saltation coefficient, $c_{0}$, are two important parameters in saltation models often assumed to be deterministic. As saltation is turbulent in nature, we argue that it is more reasonable to consider them as parameters obeying certain probability distributions. We estimate these distributions using the JADE data. The factors contributing to the stochasticity of $u_{* t}$ and $c_{0}$ are examined.
\end{abstract}

\section{Introduction}

It is well recognised that saltation, the hopping motion of sand grains near the Earth's surface, is a turbulent process (Bagnold, 1941). However, early studies focused mainly on its mean behaviour. The most well known is the Owen (Owen, 1964) saltation model, which predicts that the vertically integrated saltation flux is proportional to $u_{*}$ cubed, where $u_{*}$ is friction velocity, defined as $u_{*}=\sqrt{\tau / \rho}$ with $\tau$ being surface shear stress $\left(\mathrm{N} \mathrm{m}^{-2}\right)$ and $\rho$ being air density $\left(\mathrm{kg} \mathrm{m}^{-3}\right)$. A dedicated investigation on turbulent saltation was conducted by Butterfield (1991), which revealed the significant variability of saltation fluxes concealed in conventional time-averaged data. Stout and Zobeck (1997) introduced the idea of saltation intermittency and pointed out that even when the averaged $u_{*}$ is below the threshold friction velocity, $u_{* t}$, saltation can still intermittently occur. The latter authors emphasized saltation intermittency caused by fluctuations of turbulent wind, but stochasticity of $u_{* t}$ can also play a role. Turbulent saltation has attracted much attention in more recent years (e.g. McKenna-Neuman et al., 2000; Davidson-Arnott and Bauer, 2009; Sherman et al., 2017) and large-eddy simulation models have been under development to model the process (e.g. Dupont et al., 2013). However, due to a lack of high-frequency field observations of saltation fluxes, the statistical behaviour of turbulent saltation is to date not well understood.

A related problem is how saltation can be parameterised in wind erosion models. For example, for dust modelling, it is important to quantify saltation, as saltation bombardment is the main mechanism for dust emission. In wind erosion models, $u_{* t}$ is a key parameter which depends on many factors including soil texture, moisture, salt concentration, crust and surface roughness. In models, it is often expressed as

$u_{* t}\left(d ; \lambda, \theta, s_{l}, c_{r}, \ldots\right)=u_{* t}(d) f_{\lambda}(\lambda) f_{\theta}(\theta) f_{s l}\left(s_{l}\right) f_{c r}\left(c_{r}\right) \ldots$

where $u_{* t}(d)$ is the minimal threshold friction velocity for grain size $d$ (Shao and $\mathrm{Lu}, 2000$ ), $\lambda$ is roughness frontalarea index, $\theta$ is soil moisture, $s_{l}$ is soil salt content and $c_{r}$ is a descriptor of surface crustiness. $f_{\lambda}, f_{\theta}, f_{\mathrm{s} l}$ and $f_{\mathrm{c} r}$ are the corresponding correction functions. The corrections are determined semi-empirically, e.g. $f_{\lambda}$ using the Raupach et 
al. (1993) scheme and $f_{\theta}$ the Fécan et al. (1999) scheme. The corrections $f_{\mathrm{s} l}$ and $f_{\mathrm{cr}}$ are so far not well known.

For homogeneous saltation, the saltation flux can be computed using the Kawamura (1964) scheme, multiplied here by the fraction of erodible surface area $\sigma_{f}$,

$$
Q(d)= \begin{cases}\sigma_{f} c_{0} \frac{\rho}{g} u_{*}^{3}\left(1-\frac{u_{* t}}{u_{*}}\right)\left(1+\frac{u_{* t}}{u_{*}}\right)^{2} & u_{*}>u_{* t} \\ 0 & u_{*} \leq u_{* t}\end{cases}
$$

where $d$ is the particle diameter in a sand particle size range and $g$ is acceleration due to gravity. The saltation coefficient, $c_{0}$, is usually estimated empirically from field and/or wind tunnel experiments. It falls between 1.8 and 3.1 according to Kawamura (1964), and is commonly set to 2.6 (White, 1979) in wind erosion models. The total (all particle sizes) saltation flux, $Q$, is a particle-size weighted average of $Q(d)$

$$
Q=\int_{d_{1}}^{d_{2}} Q(d) p_{\mathrm{s}}(d) \delta \mathrm{d}
$$

where $d_{1}$ and $d_{2}$ define the upper and lower limits of saltation particle size respectively, and $p_{\mathrm{s}}(d)$ is the soil particle size distribution. Observations show, however, $c_{0}$ varies considerably from case to case (e.g. Gillette et al., 1997; Leys, 1998), and as the data presented later in this paper show, for a given location, it may vary from day to day and even during a wind erosion event.

While wind erosion modules built into numerical weather and global climate models (e.g. Shao et al., 2011; Kok et al., 2014; Klose et al., 2014) are in general more sophisticated than what is described above and include a dust emission scheme, the estimate of $Q$ is essentially done using Eqs. (1) to (3) or similar. Thus, the estimates of $u_{* t}$ and specification of $c_{0}$ are critical to wind erosion and dust modelling.

In most wind erosion models, both $u_{* t}$ and $c_{0}$ are treated as being deterministic. As saltation is turbulent, it is more rational to treat $u_{* t}$ and $c_{0}$ as parameters that satisfy certain probability distributions. Saltation intermittency also implies that $u_{* t}$ and $c_{0}$ depend on the scale of averaging. Shao and Mikami (2005) noticed that $u_{* t}$ for $10 \mathrm{~min}$ averaged $Q$ and 1 min averaged $Q$ are quite different. Namikas et al. (2003) and Ellis et al. (2012) have also noticed that averaging intervals of surface shear stress are important to quantifying sediment transport because both shear stress and saltation flux are turbulent.

Between 23 February and 14 March 2006, Ishizuka et al. $(2008,2014)$ carried out the Japan-Australia Dust Experiment (JADE) in Australia. In JADE, both $u_{*}$ and $Q$, together with a range of atmospheric and soil surface quantities, were measured at relatively high sampling rates. The loamy sand soil surface at the JADE site was very mobile and thus the JADE data are representative of surfaces that are almost ideal for sand drifting. In this study, we analyse some aspects of the turbulent behaviour of saltation using the JADE measurements of saltation fluxes. In light of the analysis, we ask the question of what the most likely values of $u_{* t}$ and $c_{0}$ are and how representative they are. We also estimate the probability distribution of the two parameters.

\section{Data and method for parameter estimation}

\subsection{JADE data}

Ishizuka et al. carried out JADE between 23 February and 14 March 2006 on an Australian farm at $\left(33^{\circ} 50^{\prime} 42.4^{\prime \prime} \mathrm{S}\right.$, $142^{\circ} 44^{\prime} 9.0^{\prime \prime} \mathrm{E}$ ). The size of field is about $1 \mathrm{~km}$ in the E-W direction and about $4 \mathrm{~km}$ in the $\mathrm{N}-\mathrm{S}$ direction. A range of atmospheric variables, land surface properties, soil particlesize distributions and size-resolved sand and dust fluxes were measured. During the study period, 12 wind erosion episodes were recorded. The data set is particularly valuable in that particle-size-resolved sand and dust fluxes (Shao et al., 2011) were measured. The details of the experiments and data sets can be found in Ishizuka et al. $(2008,2014)$ and hence only a brief summary is given here.

In JADE, three sand particle counters (SPCs) (Yamada et al., 2002) were used to measure saltation at the $0.05,0.1$ and $0.3 \mathrm{~m}$ levels with a sampling rate of $1 \mathrm{~Hz}$. A SLD (super luminescent diode) light source is used to detect particles flying through the light beam. The frequency of the input signal is $1-30 \mathrm{kHz}$, implying that particles moving at a speed of less than $30 \mathrm{~m} \mathrm{~s}^{-1}$ can be detected. A SPC measures the saltation of particles in the range of 39-654 $\mu \mathrm{m}$ in 32 bins with mean diameters of $39,54,69 \mu \mathrm{m}$ etc. with irregular increments ranging between 15 and $23 \mu \mathrm{m}$. At each measurement height, the saltation flux density $\left(\mathrm{ML}^{-2} \mathrm{~T}^{-1}\right), q$, is obtained as the sum of $q_{j}$ (saltation flux for size bin $j$ ) for the 32 size bins, i.e.

$q=\sum_{j=1}^{32} q_{j}$

The saltation flux, $Q$, is then estimated by integrating $q$ over height, namely

$Q=\int q d z$.

In computing $Q$, we assume $q=q_{0} \exp (-a z)$ with $q_{0}$ and $a$ being fitting parameters from the measurements. Prior to the field experiment, the SPCs were calibrated in the laboratory and during JADE. They were checked in a mobile wind tunnel at the site and compared with other saltation samplers. But as $q$ was measured only at three heights, the vertical resolution of $q$ is relatively poor and inaccuracies in the $Q$ estimates are unavoidable, which we are unable to fully quantify. However, the profiles of $q$ are well behaved and thus the inaccuracies in the absolute values of the $Q$ estimates are not 
expected to be so large as to affect the conclusions of this study.

$Q$ is computed using the SPC data at $1 \mathrm{~s}$ intervals. We denote its time series by $Q_{1 \mathrm{~s}}$. From $Q_{1 \mathrm{~s}}$, the $1 \mathrm{~min}$ averages, $Q_{1 \mathrm{~min}}$, and $30 \mathrm{~min}$ averages of saltation fluxes, $Q_{30 \mathrm{~min}}$, are derived (Ishizuka, 2018). All these quantities are also computed for individual particle size bins as

$Q_{j}=\int q_{j} \mathrm{~d} z$

Atmospheric variables, including wind speed, air temperature and humidity at various levels, as well as radiation, precipitation, soil temperature and soil moisture were measured using an automatic weather station (AWS). These quantities were sampled at $5 \mathrm{~s}$ intervals and their averages over $1 \mathrm{~min}$ intervals were recorded. Two anemometers were mounted at heights of 0.53 and $2.16 \mathrm{~m}$ on a mast for measuring wind speed. Also available are the Monin-Obukhov length and sensible heat fluxes. From the wind measurements, surface roughness length $z_{0}$ and friction velocity $u_{*}$ are derived, assuming a logarithmic profile (with stability correction) of the mean wind. The roughness length for the experimental site is estimated to be $0.48 \mathrm{~mm}$.

Friction velocity is computed with $1 \mathrm{~min}$ averaged wind data, denoted by $u_{* 1 \mathrm{~min}}$, and $30 \mathrm{~min}$ averaged wind data, denoted by $u_{* 30 \mathrm{~min}}$ (Ishizuka, 2018). In atmospheric boundarylayer studies, there is no standard for the length of time over which one should average wind to correctly estimate $u_{*}$, but it is common to average it over 10 to $30 \mathrm{~min}$. But the time period depends on the purpose of the averaging. If $u_{*}$ is used as a scaling velocity for the atmospheric boundary layer, e.g. as measure of turbulence intensity, it is necessary to average over a sufficiently large time interval to obtain a constant $u_{*}$. In this paper, $u_{*}$ is a surrogate of shear stress, the variation of which drives that of saltation. Therefore, short averaging times are preferred, subject to them being larger than the response time of aeolian flux to shear stress. Anderson and Haff (1988) and Butterfield (1991) suggested that this response time is of the order of $1 \mathrm{~s}$.

Observations of surface soil properties, including soil temperature and soil moisture, were made at 1 min intervals. The surface at the JADE site was relatively uniform. A survey of ground cover over an area of $900 \times 900 \mathrm{~m}^{2}$ at the site was made on 11 March 2006. The area was divided into nine tiles and surveyed along one transect of $300 \mathrm{~m}$ long in each tile. Photographs were taken every $5 \mathrm{~m}$ by looking down vertically to a point on the ground. Surface cover was estimated to be $\sim 0.02$ (see Appendix of Shao et al., 2011).

The wind erosion model, as detailed in Shao et al. (2011), is used for computing the saltation fluxes using the JADE atmospheric and surface soil measurements as input. The saltation model component is as described in Section 1, consisting of Eqs. (1)-(3). The fraction of erodible surface area, $\sigma_{\mathrm{f}}$, used in Eq. (2), is 1 minus the fraction of surface cover. The soil particle size distribution (psd), $p_{\mathrm{s}}(d)$, required for Eq. (3), is based on soil samples collected at the JADE site and analysed in the laboratory. The analysis was done using a Microtrac (Microtrac MT3300EX, Nikkiso Co. Ltd.), a particle size analyser based on laser diffraction light-scattering technology. Water was used for sample dispersion. Depending on the methods (pretreatment and ultrasonic vibration) used, the soil texture can be classified as sandy loam (clay $0.3 \%$, silt $25 \%$ and sand $74.7 \%$ ) or loamy sand (clay $11 \%$, silt $35 \%$ and sand $54 \%$ ). The sandy loam psd is used in this study, which has a mode at $\sim 180 \mu \mathrm{m}$ (see Shao et al., 2011, Fig. 5, Method A).

The default value of $c_{0}$ is set to 2.6, as widely cited in the literature (e.g. White, 1979) and the default value of $u_{* t}$ is computed using Eq. (1) with $u_{* t}(d)$ computed using the Shao and Lu (2000) scheme, $f_{\lambda}$ using the Raupach et al. (1993) scheme, $f_{\theta}$ the Fécan et al. (1999) scheme, and $f_{\mathrm{s} l}$ and $f_{\mathrm{c} r}$ set to one. The frontal area index $\lambda$ and soil moisture $\theta$ are both observed data from JADE.

\subsection{Method for parameter estimation}

Different choices of $c_{0}$ and $u_{* t}$ would lead to different model-simulated saltation fluxes which may or may not agree well with the measurements. By fitting the simulated saltation fluxes to the measurements, we determine the optimal estimates of $c_{0}$ and $u_{* t}$ and the probability density function (PDF) of these parameters. The method based on the Bayesian theory is used for this purpose.

Suppose $\widetilde{X}=\left(\widetilde{x}_{1}, \widetilde{x}_{2}, \ldots, \widetilde{x}_{n}\right)$ is a measurement vector, with $\widetilde{x}_{i}$ being the measured value at time $t_{i}$, and $A$ is a model with a forcing vector $F$ and model parameter vector $\beta$. Let the initial state of the system be $i_{0}$, so the modelled value of the system, $X=\left(x_{1}, x_{2}, \ldots, x_{n}\right)$, can be expressed as

$X(\beta)=A\left(i_{0}, F ; \beta\right)$

The error vector is given by $E(\beta)=\widetilde{X}-X$, here and is fully attributed to $\beta$. Given $\widetilde{X}$, the posterior parameter PDF, $p(\beta \mid \widetilde{X})$, can be estimated from the Bayes theorem:

$p(\beta \mid \tilde{X}) \propto p(\beta) p(\widetilde{X} \mid \beta)$,

where $p(\beta)$ is the prior parameter $\operatorname{PDF}$ and $p(\widetilde{X} \mid \beta)$ is the likelihood. If $p(\beta)$ is given, then the problem of finding $p(\beta \mid \tilde{X})$ reduces to finding the maximum likelihood. Assuming the error residuals are independent and Gaussian distributed with constant variance, $\sigma^{2}$, the likelihood can be written as

$p(\tilde{X} \mid \beta)=\prod_{i=1}^{n} \frac{1}{\sqrt{2 \pi} \sigma} \exp \left(-\frac{\left(x_{i}-\tilde{x}_{i}\right)^{2}}{2 \sigma^{2}}\right)$.

In this case, maximising the likelihood is equivalent to minimising the error, i.e.

$R^{2}(\beta)=\min \sum_{i}\left(x_{i}-\tilde{x}_{i}\right)^{2}$. 

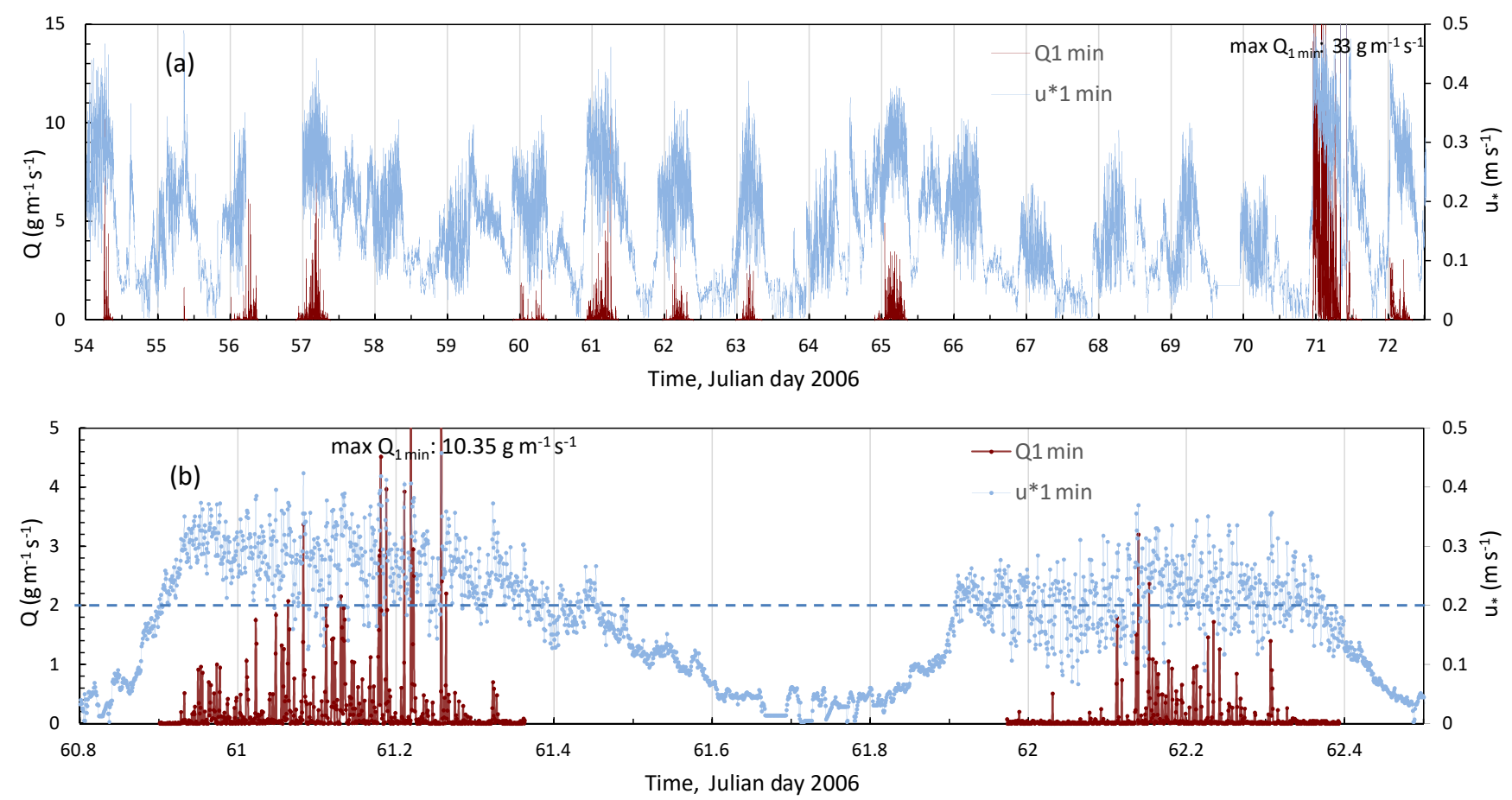

Figure 1. (a) Observed time series of 1 min averaged saltation flux, $Q_{1 \mathrm{~min}}\left(\mathrm{~g} \mathrm{~m}^{-1} \mathrm{~s}^{-1}\right)$, and friction velocity, $u_{* 1} \mathrm{~min}\left(\mathrm{~m} \mathrm{~s}{ }^{-1}\right)$, for the JADE study period; (b) an enlarged plot of (a) for the erosion events on days 61 and 62. Note that the axes in (b) have different scales than in (a).

The solution of Eq. (10) gives an optimal (i.e. with maximum likelihood) estimate of mean $\beta$. This is the popular least squares method. A disadvantage of the method is that it assumes a Gaussian posterior parameter PDF and computing the $\beta$ variance requires prior knowledge of the accuracy of the data.

As an alternative, the approximate Bayesian computation (ABC) method has been proposed (e.g. Vrugt and Sadegh, 2013). It is argued that a parameter value $\beta^{*}$ should be a sample of $p(\beta \mid \widetilde{X})$ as long as the distance between the observed and simulated data is less than a small positive value

$\rho\left(\beta^{*}\right)=\left|X\left(\beta^{*}\right)-\tilde{X}\right| \leq \varepsilon$.

This procedure explicitly provides an estimate of parameter PDF for a given data set. The ABC method is numerically simple: from a prior PDF (e.g. uniform) of $\beta$ a $\beta^{*}$ is stochastically generated and the model is run. If Eq. (10) is satisfied, then $\beta^{*}$ is accepted or otherwise rejected. This procedure is repeated and the a priori PDF of $\beta$ is mapped to a posterior $\mathrm{PDF}$ of $\beta$. The $\mathrm{ABC}$ method has the disadvantage that it is numerically inefficient. More efficient techniques based on the same principle exist, e.g. the Markov chain Monte Carlo simulation (Sadegh and Vrugt, 2014). In this study, we apply the Differential Evolution Adaptive Metropolis (DREAM) algorithm proposed by Vrugt et al. (2011) for estimation of hydrologic model parameters. The algorithm integrates differential evolution (Storn and Price, 1997) and self-adaptive randomised subspace sampling to accelerate a Markov chain
Monte Carlo simulation. A full description of the DREAM algorithm is beyond the scope of our study. Interested readers should refer to the above-cited references for details.

\section{Statistical features of saltation}

\subsection{Time series}

To provide an overview of the data set used in this study, Fig. 1 shows the time series of $Q_{1 \mathrm{~min}}$ and $u_{* 1 \mathrm{~min}}$, and Fig. 2, $Q_{30 \mathrm{~min}}$ and $u_{* 30 \mathrm{~min}}$. During the 20-day period, aeolian sand drift occurred almost every day at the site according to the field logging book, but only 12 events were recorded using the SPCs. Saltation fluxes were not measured on days 55,58 , 59,64 and then days 66 to 70 due to either instrument maintenance or use of the SPCs for other purposes (e.g. wind tunnel experiments). The figures show that both $Q$ and $u_{*}$ fluctuate significantly and saltation is turbulent. Figure $1 \mathrm{~b}$ shows an enlarged plot of the $Q_{1 \mathrm{~min}}$ and $u_{* 1 \mathrm{~min}}$ time series for days 61 and 62 . At the JADE site, $u_{* t}$ was about $0.2 \mathrm{~m} \mathrm{~s}^{-1}$. On day $61, u_{*}$ was mostly larger than this value and saltation was almost continuous, while on day $62, u_{*}$ was close to this value and weak saltation occurred frequently also when $u_{*}$ was below $0.2 \mathrm{~m} \mathrm{~s}^{-1}$. Figure $2 \mathrm{~b}$ is as Fig. $1 \mathrm{~b}$ but for $Q_{30 \mathrm{~min}}$ and $u_{* 30 \mathrm{~min}}$. A comparison of Figs. $1 \mathrm{~b}$ and $2 \mathrm{~b}$ reveals that the amplitude of the $Q_{1 \text { min }}$ fluctuations is several times of that of the $Q_{30 \text { min }}$ fluctuations. A strong correlation between the 

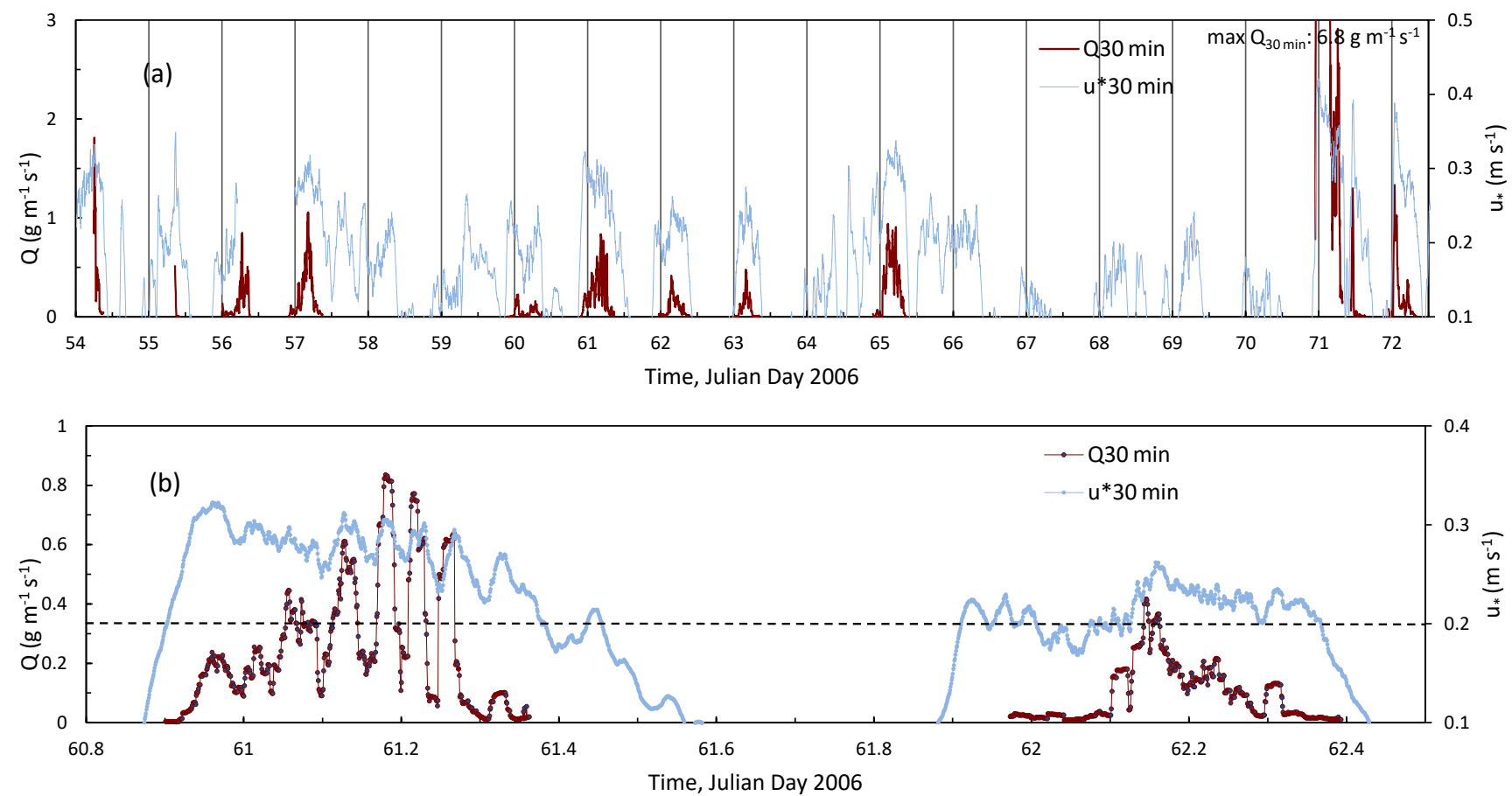

Figure 2. As Fig. 1 but for running means over 30 min intervals.

time series of $Q_{30 \min }$ and $u_{* 30 \text { min }}$ can be directly seen in Fig. 2b.

In Fig. 3a, b and c, $Q$ is plotted against $u_{*}^{3}$. Several interesting features can be identified. For the majority of the points, the $Q \sim u_{*}^{3}$ relationship appears to hold, but this relationship can vary significantly even for the same data set from event to event. For example, large differences exist between days 70 and 71 (denoted by D70-71, an event of intensive wind erosion) and day 72 (a day of weak wind erosion), as seen in both Fig. 3a and b. There may be many likely reasons for the differences in the $Q \sim u_{*}$ relationship but the most conspicuous are differences in atmospheric turbulence (e.g. gustiness) and time-varying surface conditions (e.g. particle sorting and aerodynamic roughness). Figure $3 \mathrm{~d}$ shows the time series of $\left(u_{* 1} \min -u_{* 30 \mathrm{~min}}\right)$, a measure of turbulent fluctuations. It is seen that saltation is associated with not only high surface shear stress but also high shear stress fluctuations. The large difference in the $Q \sim u_{*}$ relationship between D70-71 and D72 (Fig. 3b) is probably attributed to the strong differences in turbulent fluctuations (Fig. 3d): D70-71 was a hot gusty day with top $(2 \mathrm{~cm})$ soil temperature reaching $53{ }^{\circ} \mathrm{C}$, while D72 was cooler and less gusty with soil temperature $5{ }^{\circ} \mathrm{C}$ lower. Also hysteresis is observed in the $Q \sim u_{*}$ relationship, as shown in Fig. 3c, using D71 and D72 as examples. Figure $3 \mathrm{~d}$ shows that for all three events selected (D70-71, D71 and D72), saltation has a relatively short ( 0.5 to $2 \mathrm{~h}$ ) strengthening phase, followed by a longer weakening phase. During an erosion event for the same $u_{*}$, saltation is stronger in the strengthening phase than in the weakening phase. An exam- ination of Fig. 3d suggests that the hysteresis cannot simply be attributed to the intensity of turbulence. We speculate that it is probably more related to flow-saltation feedbacks (e.g. stronger splash entrainment in the strengthening phase) and the modification of surface aerodynamic conditions (e.g. particle sorting and reduced surface roughness Reynolds number).

\subsection{Probability density function of saltation fluxes}

How well the saltation model performs, whether $u_{* t}$ and $c_{0}$ are universal and how they are probabilistically distributed must depend on the turbulent properties of saltation. As the JADE saltation fluxes are sampled at $1 \mathrm{~Hz}$, we can use these data to examine (to some degree) the statistical behaviour of saltation. In Fig. 4, the PDFs of the saltation fluxes for different particle size groups are plotted and computed using $Q_{1 \text { s }}$ and $Q_{1 \text { min. }}$. It is seen that the PDFs generally behave as

$p(Q) \propto Q^{-\alpha}$.

In the case of $Q_{1 \mathrm{~s}}$, there seems to be a distinct change in $\alpha$ at a critical value of $Q_{\mathrm{c}} \sim 3 \mathrm{~g} \mathrm{~m}^{-1} \mathrm{~s}^{-1}$, with $\alpha \sim 1$ for $Q<Q_{\mathrm{c}}$ and $\alpha \sim 4$ for $Q>Q_{\text {c }}$. The PDFs derived from $Q_{1 \text { min appear }}$ to follow the basic functional form of Eq. (11). Again, $\alpha$ is about 1 and tends to be larger for large $Q$ values. Figure 4 shows that the PDFs of $Q$ depend significantly on the interval of time averaging; i.e. after averaging, smaller saltation fluxes become more frequent. This is because the time series of $Q_{1 \mathrm{~s}}$ is more intermittent (see also Fig. 6). 

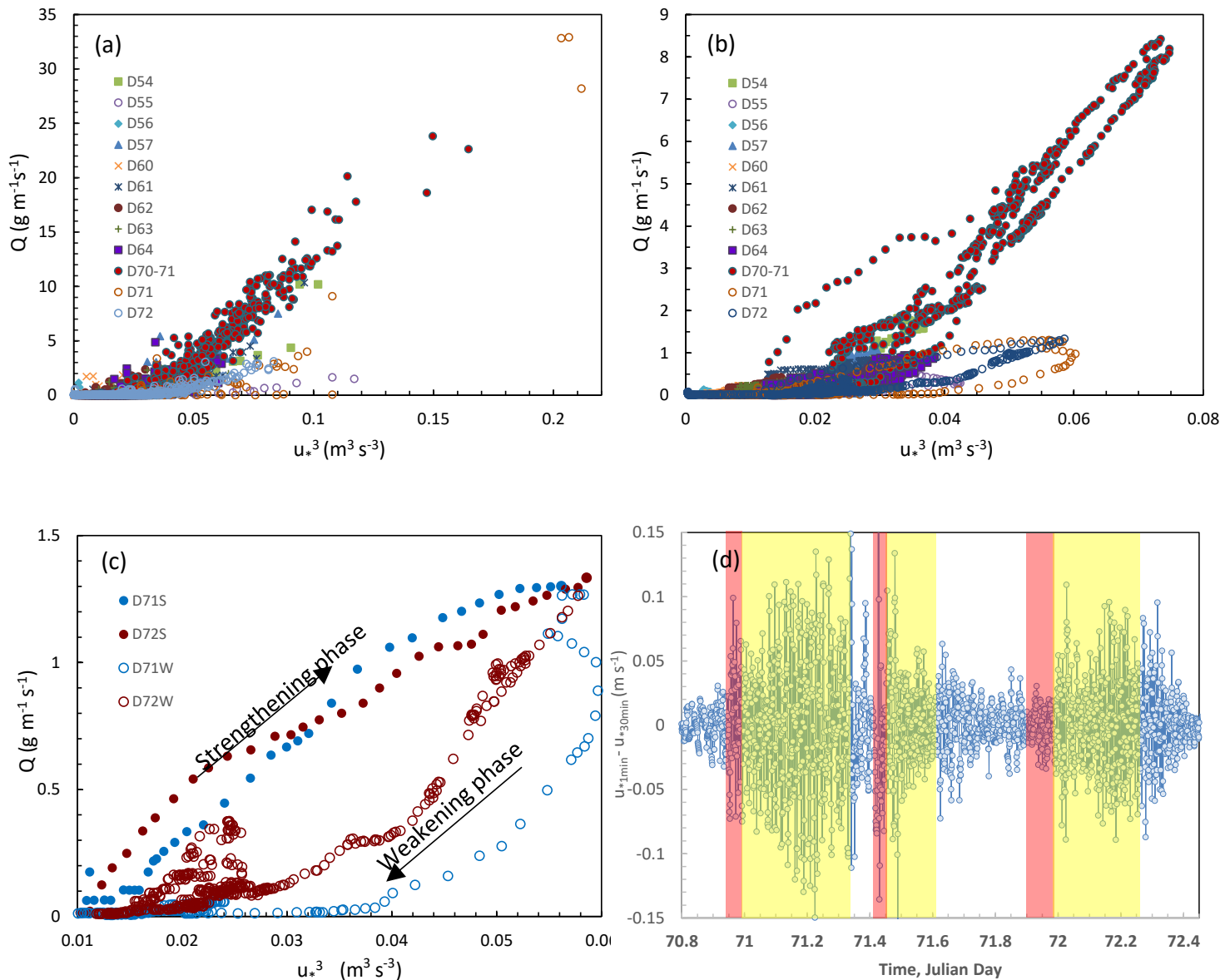

Figure 3. (a) Saltation flux, $Q\left(\mathrm{~g} \mathrm{~m}^{-1} \mathrm{~s}^{-1}\right)$, plotted against friction velocity, $u_{*}^{3}\left(\mathrm{~m}^{3} \mathrm{~s}^{-3}\right)$, for 1 min averages. (b) As (a) but for 30 min averages. (c) As (b) but enlarged to illustrated saltation hysteresis on D71 and 72; D71S/72S denotes the strengthening phase and D71W/72W the weakening phase of the D71/72 event. (d) Time series of $u_{*}$ derivations, given by $\left(u_{* 1 \mathrm{~min}}-u_{* 30 \mathrm{~min}}\right)$, for D70-71, D71 and D72. The strengthening phase is marked red and the weakening phase is in yellow.

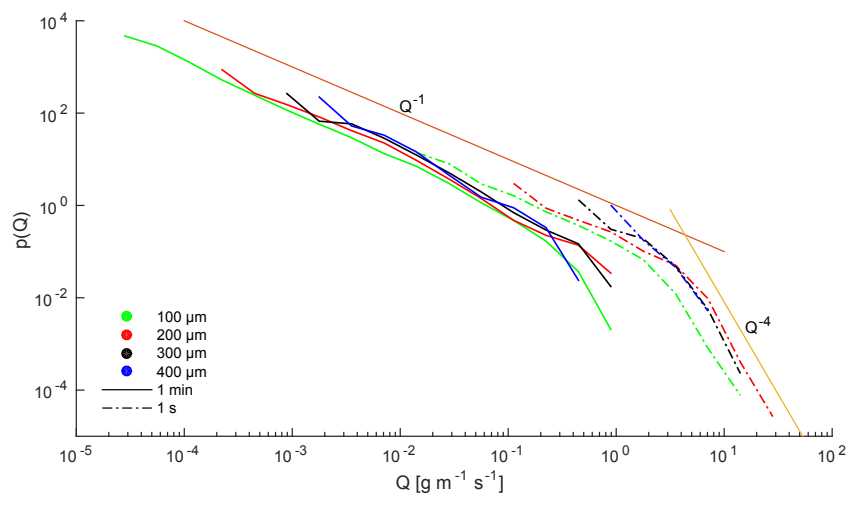

Figure 4. Probability density functions of $Q_{1}$ s (solid lines) and of $Q_{1 \text { min }}$ (dashed lines) for four different particle sizes. Two additional lines $p(Q) \sim Q^{-1}$ and $Q^{-4}$ are drawn for reference.
The PDFs of $Q_{1 \mathrm{~s}}$ and $Q_{1 \text { min }}$ integrated over all particles are shown in Fig. 5b. Again, the PDFs show the general behaviour of $p(Q) \sim Q^{-1}$. In theory, $p(Q)$ can be derived from the PDF of $u_{*}, p\left(u_{*}\right)$. From Eq. (2), we have

$\frac{d Q}{d u_{*}}=c_{0} \frac{\rho}{g}\left(3 u_{*}^{2}+2 u_{*} u_{* t}-u_{* t}^{2}\right) \quad$ for $\quad u_{*}>u_{* t}$.

This can be used to obtain

$p(Q)=\left\{\begin{array}{lll}p\left(u_{*}\right) \frac{d u_{*}}{d Q} & \text { for } & u_{*}>u_{* t} \\ 0 & \text { for } & u_{*} \leq u_{* t}\end{array}\right.$

Figure 5a shows the $p\left(u_{*}\right)$ estimated from $u_{* 1 \text { min }}$ together with the fitted Weibull distribution. For the fitting, we ensure that $p\left(u_{*}\right)$ for $u_{*}>0.2 \mathrm{~m} \mathrm{~s}^{-1}$ is best approximated. Figure $5 \mathrm{~b}$ shows the $p(Q)$ estimated from $Q_{1 \mathrm{~min}}$. We computed $p(Q)$ using Eq. (13) with the fitted $p\left(u_{*}\right)$, assuming $u_{* t}=0.2 \mathrm{~m} \mathrm{~s}^{-1}$ and $c_{0}=2.6$. It is seen that the observed and modelled $p(Q)$ have qualitative similarities but using 

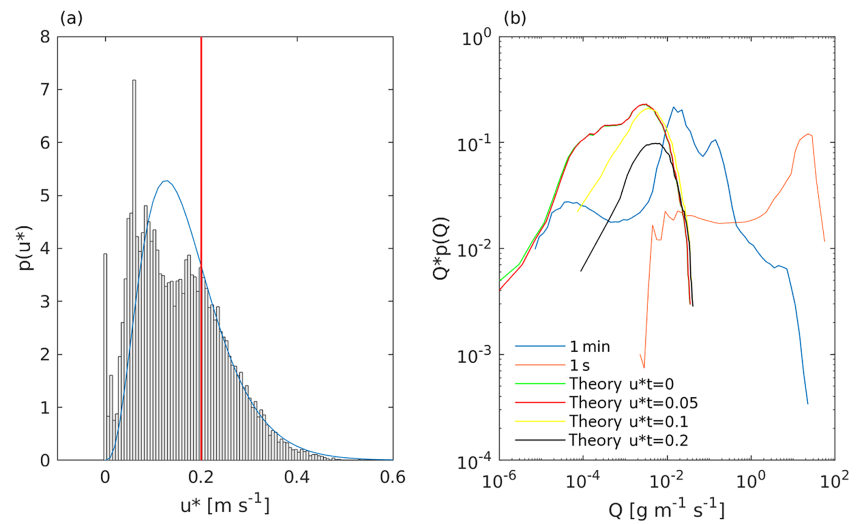

Figure 5. (a) Probability density functions of friction velocity, $p\left(u_{*}\right)$, plotted against $u_{*}$ (bars). To compute $p\left(u_{*}\right), u_{* 1 \mathrm{~min}}$ is used; a Weibull distribution (blue line) is fitted to $p\left(u_{*}\right)$; the red line marks the assumed threshold friction velocity. (b) Probability density function of $Q$, in $Q \cdot p(Q)$, estimated using $Q_{1 \text { min }}$ (blue) and $Q_{1 \mathrm{~s}}$ (dark red) and using Eq. (13) assuming several $u_{* t}$ values $\left(u_{* t}=0.0 \mathrm{~m} \mathrm{~s}^{-1}\right.$, green; $0.05 \mathrm{~m} \mathrm{~s}^{-1}$, red; $0.1 \mathrm{~m} \mathrm{~s}^{-1}$, yellow; $0.2 \mathrm{~m} \mathrm{~s}^{-1}$, black).

Eqs. (12) and (13) we cannot reproduce the observed $p(Q)$ well. For example, the model fails to predict the low frequent strong saltation fluxes and the mode of saltation fluxes. Tests using several smaller $u_{* t}$ values $(0,0.05$ and 0.1$)$ are also carried out. With smaller $u_{* t}$ values, the mode of the predicted saltation fluxes is shifted to smaller values, but the predictions are far from satisfactory.

\subsection{Saltation intermittency}

Following Stout and Zobeck (1997), the intermittency of saltation, $\gamma$, is defined as the fraction of time during which saltation occurs at a given point in a given time period. It should be pointed out that saltation intermittency describes only the behaviour of the process at $u_{*} \sim u_{* t}$; i.e. saltation intermittency is merely a special case of turbulent saltation. Several formulations of $\gamma$ are possible. Stout and Zobeck (1997) assumed that saltation occurs only in time windows in which $u_{*}$ exceeds $u_{* t}$. Therefore, if $p\left(u_{*}\right)$ is known, then $\gamma$ for a given $u_{* t}$ can be estimated as

$\gamma_{a}\left(u_{* t}\right)=1-\int_{0}^{u_{* t}} p\left(u_{*}\right) d u_{*}$.

Stout and Zobeck (1997) used the counts per second of sand impacts on a piezoelectric crystal saltation sensor as a measure of saltation activity and found that $\gamma_{a}$ rarely exceeded 0.5 .

In Eq. (14a) $u_{* t}$ is fixed and thus saltation intermittency is attributed entirely to the fluctuations of $u_{*}$. In reality, $u_{* t}$ also fluctuates and satisfies certain PDFs (Raffaele et al., 2016).
In analogy to Eq. (14a), $\gamma$ for a given $u_{*}$ can be estimated as

$\gamma_{b}\left(u_{*}\right)=1-\int_{u_{*}}^{\infty} p\left(u_{* t}\right) d u_{* t}$.

More generally, we can define saltation intermittency as

$$
\begin{aligned}
\gamma_{c} & =\int_{0}^{\infty}\left[1-\int_{0}^{u_{* t}} p\left(u_{*}\right) d u_{*}\right] p\left(u_{* t}\right) d u_{* t} \\
& =\int_{0}^{\infty} \gamma_{a}\left(u_{* t}\right) p\left(u_{* t}\right) d u_{* t},
\end{aligned}
$$

or

$$
\begin{aligned}
\gamma_{c} & =\int_{0}^{\infty}\left[1-\int_{u_{*}}^{\infty} p\left(u_{* t}\right) d u_{* t}\right] p\left(u_{*}\right) d u_{*} \\
& =\int_{0}^{\infty} \gamma_{b}\left(u_{*}\right) p\left(u_{*}\right) d u_{*} .
\end{aligned}
$$

Equations $(14 \mathrm{c})$ and $(14 \mathrm{~d})$ reduce to Eq. (14a) if $p\left(u_{* t}\right)=$ $\delta\left(u_{* t}\right)$ and to Eq. $(14 \mathrm{~b})$ if $p\left(u_{*}\right)=\delta\left(u_{*}\right)$.

The computation of saltation intermittency function $\gamma_{a}\left(u_{* t}\right)$ is done by integrating $p\left(u_{*}\right)$ (Fig. 5a) to fixed value of $u_{* t}$. In Fig. $6 \mathrm{a}, \gamma_{a}$ as a function of $u_{* t}$ is plotted. The behaviour of $\gamma_{a}\left(u_{* t}\right)$ is as expected: it is one at $u_{* t}=0$ and decreases to zero at about $u_{* t}=0.5 \mathrm{~m} \mathrm{~s}^{-1}$ as in the case of JADE, $u_{*}$ rarely exceeded this value. For $u_{* t}=0.2 \mathrm{~m} \mathrm{~s}^{-1}, \gamma_{a}$ is 0.35 , comparable with the result of Stout and Zobeck (1997) who reported an intermittency of 0.4 . As $p\left(u_{* t}\right)$ is not known, Eq. (14b) cannot be used directly, but we can compute $\gamma_{b}\left(u_{*}\right)$ using the JADE data. First, it is computed using $Q_{1 \mathrm{~min}}$. This is done by selecting a fixed $u_{*}$ say $u_{* c}$, and counting the time fraction, $T_{u *}$, which satisfies $\left|u_{*}-u_{* c}\right|<\varepsilon$ (used is $\varepsilon=0.05 \mathrm{~m} \mathrm{~s}^{-1}$ ) and the time fraction, $T_{Q 1 \min }$, which satisfies $\left|u_{*}-u_{* c}\right|<\varepsilon$ and $Q_{1 \min }>0$. By definition, saltation intermittency is $T_{Q 1 \mathrm{~min}} / T_{u *}$ as plotted in Fig. 6a. It is seen that for $Q_{1 \mathrm{~min}}, \gamma_{b}\left(u_{*}\right)$ increases from about 0.6 at $u_{*} \sim 0.1 \mathrm{~m} \mathrm{~s}^{-1}$ to about one at $u_{*}=0.3 \mathrm{~m} \mathrm{~s}^{-1}$. This shows that in JADE a considerable fraction of the saltation fluxes was recorded at $u_{*}$ below the perceived threshold friction velocity (about $0.2 \mathrm{~m} \mathrm{~s}^{-1}$ ), saltation is more intermit under weak wind conditions and becomes non-intermittent for $u_{*}>0.3 \mathrm{~m} \mathrm{~s}^{-1}$. The increase in $\gamma_{b}\left(u_{*}\right)$ with decreasing $u_{*}$ for $u_{*}<0.1 \mathrm{~m} \mathrm{~s}^{-1}$ is, however, unexpected. The expected $\gamma_{b}\left(u_{*}\right)$ for small $u_{*}$ is as depicted using the dashed line. A likely reason for the unexpected behaviour of $\gamma_{b}\left(u_{*}\right)$ is that during a wind erosion event, grains in saltation may continue to hop even when $u_{*}$ is temporarily reduced to small values. The uncertainty in the data also needs to be considered, as the sample size for determining the ratio $T_{Q 1 \mathrm{~min}} / T_{u *}$ becomes smaller. More complete data sets are required to answer these questions. Finally, $\gamma_{c}$ is computed by using Eq. (14d) and is found to be around 0.73 . For the $1 \mathrm{~s}$ case, we cannot plot $\gamma_{b}$ as a function of $u_{*}$, because $u_{*}$ is not available at such high frequency. We computed $\gamma_{c}$ for individual particle size groups 

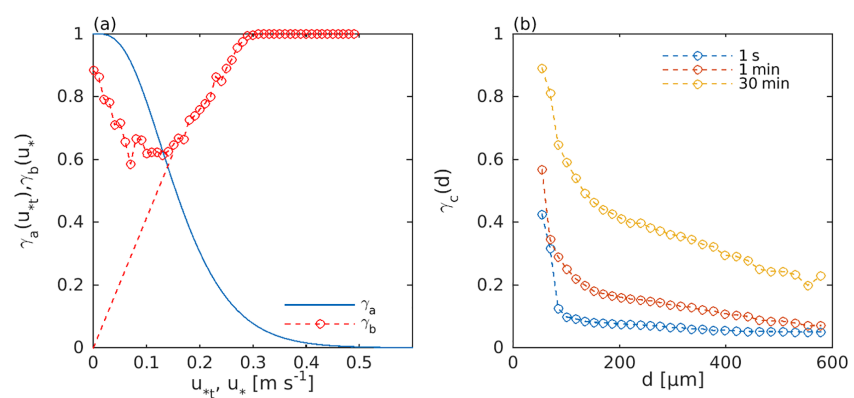

Figure 6. (a) Saltation intermittency function $\gamma_{a}\left(u_{* t}\right)$ and $\gamma_{b}\left(u_{*}\right)$. See text for more details. (b) $\gamma_{c}$ as a function of particle size for $Q_{1 \mathrm{~s}}, Q_{1 \mathrm{~min}}$ and $Q_{30 \mathrm{~min}}$.

(Fig. 6b) using $Q_{1 \mathrm{~s}}, Q_{1 \mathrm{~min}}$ and $Q_{30 \mathrm{~min}}$, which is the time fraction of saltation for a given particle size, $d$, during the saltation event. It is found that $\gamma_{c}(d)$ decreases with $d$; i.e. the saltation of larger particles is more intermittent. Also, $\gamma_{c}(d)$ increases with increased averaging time intervals, implying that the small-scale features of turbulence play an important role in intermittent saltation.

\subsection{Spectrum of saltation fluxes}

Spectral analysis is widely used for characterising the variations in a stochastic process on different scales. Using the JADE data, we computed the power spectrum of saltation fluxes, $P_{Q}(f)$ at frequency $f$, and of friction velocity, $P_{u *}(f)$, using a non-uniform discrete Fourier transform. For comparison, the power spectra are normalised with the respective variances of the signal. In atmospheric boundarylayer studies, the spectra of various turbulence quantities have been thoroughly investigated (Stull, 1988). Examples of spectra from Reynolds shear stress can be found in McNaughton and Laubach (2000). Figure 7 shows $P_{Q}(f)$ and $P_{u *}(f)$ (Fig. 7a) as well their co-spectrum (Fig. 7b). $P_{Q}(f)$ is computed using both $Q_{1 \mathrm{~s}}$ and $Q_{1 \mathrm{~min}}$, and $P_{u *}(f)$ with $u_{* 1 \mathrm{~min}}$. It is seen that the power spectra of $Q$ and $u_{*}$ have qualitatively very similar behaviour. Both have a maximum at about $10^{-5} \mathrm{~Hz}$, a minimum at about $10^{-4} \mathrm{~Hz}$ and another peak at about $2 \times 10^{-3} \mathrm{~Hz}$. The maximum at $10^{-5} \mathrm{~Hz}$ is related to the diurnal patterns and changing synoptic events, which drive the wind erosion episodes, the minimum at $10^{-4} \mathrm{~Hz}$ is due to the lack of turbulent winds at the timescale of several hours, while the peak at $2 \times 10^{-3} \mathrm{~Hz}$ is caused by the minute-scale gusty winds/large eddies in turbulent flows. Also the $Q-u_{*}$ co-spectrum shows that $Q$ and $u_{*}$ are most strongly correlated on diurnal/synoptic and gust/large-eddy timescales. $P_{Q}(f)$ computed using $Q_{1 \mathrm{~s}}$ again reveals the peaks at $10^{-5} \mathrm{~Hz}$ and at $2 \times 10^{-3} \mathrm{~Hz}$. The power of the $Q$ spectrum then decreases with frequency. As the sampling rate of saltation flux is limited to $1 \mathrm{~s}$ in this study, the features of $P_{Q}(f)$ at frequencies larger than $0.5 \mathrm{~Hz}$ are not resolved.
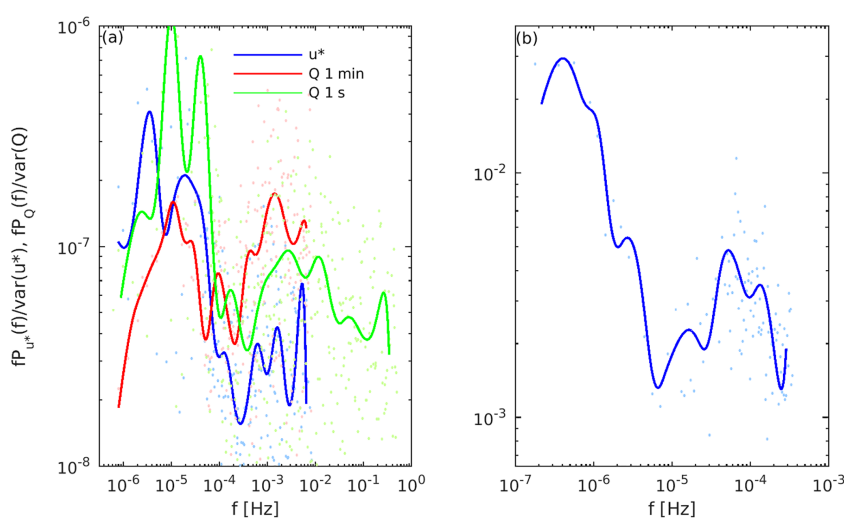

Figure 7. (a) Normalised power spectrum of $u_{*}$ (blue) computed with $u_{* 1 \mathrm{~min}}$, together with the normalised power spectrum of saltation flux computed with $Q_{1 \mathrm{~min}}$ (red) and $Q_{1 \mathrm{~s}}$ (green). (b) Normalised $Q-u_{*}$ co-spectrum, computed using with $Q_{1 \text { min }}$ and $u_{* 1}$ min. In both (a) and (b), dots are unsmoothed spectra, and curves are smoothed spectra.

\section{Estimates of saltation model parameters}

Given the turbulent nature of saltation, it is rational to treat $u_{* t}$ and $c_{0}$ in the saltation model as parameters obeying certain probability distributions. To examine the behaviour of these parameters, we introduce two coefficients $r_{c_{0}}$ and $r_{u_{* t}}$ and multiply them by the theoretical values of $c_{0}$ and $u_{* t}$, respectively, in Eq. (2), i.e.

$u_{* t}=r_{u_{* t}} u_{* t, \text { theory }}$

$c_{0}=r_{c_{0}} c_{0, \text { theory }}$.

As introduced in Sect. 1, we assumed $c_{0 \text {,theory }}=2.6$ and computed $u_{* t \text {,theory }}$ using Eq. (1) with observed soil moisture and fraction of cover. The two coefficients $r_{c_{0}}$ and $r_{u_{* t}}$ are varied to generate a model estimate of $Q$ using Eqs. (2) and (3) with observed $u_{*}$. The probability distributions of $r_{c_{0}}$ and $r_{u_{* t}}$ are estimated using the following techniques. Let us denote the time series of the modelled saltation flux by $Q_{M, i \text {, }}$ $(i=1, N)$ and the corresponding measurement by $Q_{D, i}$. The absolute error, $\delta Q_{A}$, and Nash coefficient, $I_{\text {Nash }}$, are used as measures for the goodness of the agreement between the model and the measurement. They are defined as

$\begin{aligned} \delta Q_{A} & =\frac{1}{N} \sum\left|a_{i}\right| \\ I_{\text {Nash }} & =\left(1-\sum a_{i}^{2} / \sum b_{i}^{2}\right),\end{aligned}$

with

$a_{i}=Q_{M, i}-Q_{D, i}$

$b_{i}=Q_{M, i}-\frac{1}{N} \sum Q_{M, i}$

$c_{i}= \begin{cases}a_{i} / Q_{M, i} & Q_{M, i} \neq 0 \\ 0 & \text { else. }\end{cases}$ 


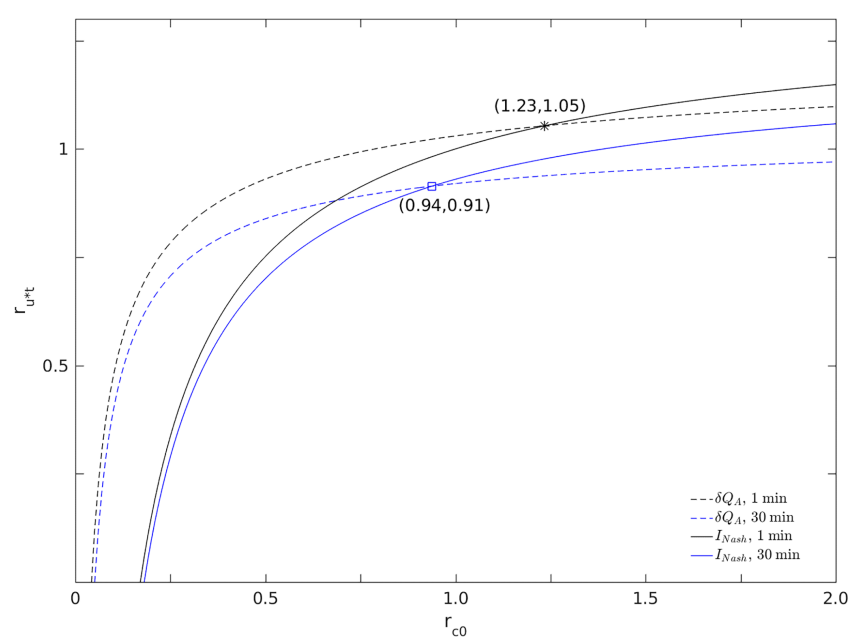

Figure 8. $\delta Q_{A}$ and $I_{\text {Nash }}$ are both functions of $r_{c_{0}}$ and $r_{u_{* t}}$. Along the dashed curves, the condition $\delta Q_{A}=\min$ is satisfied and along the solid curves the condition $I_{\mathrm{Nash}}=\max$ is satisfied. The curves are estimated with both $Q_{1 \mathrm{~min}}$ and $Q_{30 \mathrm{~min}}$.

The prior PDFs of $r_{c_{0}}$ and $r_{u_{* t}}$ are assumed to be uniform. In the numerical experiment, we randomly generate $r_{c_{0}}$ and $r_{u_{* t}}$ and seek their values, such that $\delta Q_{A} \leq \varepsilon$ and $I_{\text {Nash }}>\eta$. These experiments are repeated for $Q_{1 \mathrm{~min}}$ and $Q_{30 \mathrm{~min}}$. The plots of $\delta Q_{A}$ and $I_{\text {Nash }}$ as functions of $r_{c_{0}}$ and $r_{u * t}$ show that for certain values of $r_{c_{0}}$ and $r_{u_{*} t}$, the above conditions are satisfied. Figure 8 shows that for $Q_{1 \mathrm{~min}}$, the best simulation is achieved with $r_{c_{0}}=1.23$ and $r_{u_{* t}}=1.05$, while for the $Q_{30 \mathrm{~min}}$, with $r_{c_{0}}=0.94$ and $r_{u_{* t}}=0.91$. This suggests that the optimal estimates of $u_{* t}$ and $c_{0}$ are close to the corresponding theoretic values but are dependent on the timeaveraging intervals, with both $u_{* t}$ and $c_{0}$ being larger for shorter averaging intervals.

The parameter PDFs $p\left(r_{u_{* t}}\right)$ and $p\left(r_{c_{0}}\right)$ are estimated with the DREAM algorithm, again using the absolute error and the Nash coefficient as goodness of agreement between the model simulated and measured saltation fluxes. The results are shown in Fig. 9. All PDFs are fitted to a $\Gamma$ distribution. As seen in Fig. 9a and c, the most frequent $r_{u_{* t}}$ values are 1.12 and 1.04 for $Q_{1 \mathrm{~min}}$ and $Q_{30 \mathrm{~min}}$, close to the estimates of 1.05 and 0.91 found in Fig. 8. For $Q_{1 \mathrm{~min}}, r_{u_{* t}}$ is $\sim 1.12 \pm 0.2$ and for $Q_{30 \mathrm{~min}} \sim 1.04 \pm 0.3$. This implies that saltation sometimes occurs when $u_{*}$ is below the theoretical $u_{* t}$ value and sometimes saltation does not occur even when $u_{*}$ is above it, as already seen in Fig. 6a. In the case of $p\left(r_{c_{0}}\right)$ (Fig. 9c and d), the most frequent values of $r_{c_{0}}$ for $Q_{1 \mathrm{~min}}$ and $Q_{30 \mathrm{~min}}$ are respectively 1.04 and 0.92 , close to the optimal estimates of 1.23 and 0.94 shown in Fig. 8. But $r_{c_{0}}$ varies over a wide range, for instance, for $Q_{30}$ min between 0.5 and 5 ; i.e. $c_{0}$ is a rather stochastic parameter.

In nature, many factors influence sediment transport, but the stochasticity of the parameters is determined primarily by the turbulent fluctuations of friction velocity (or surface
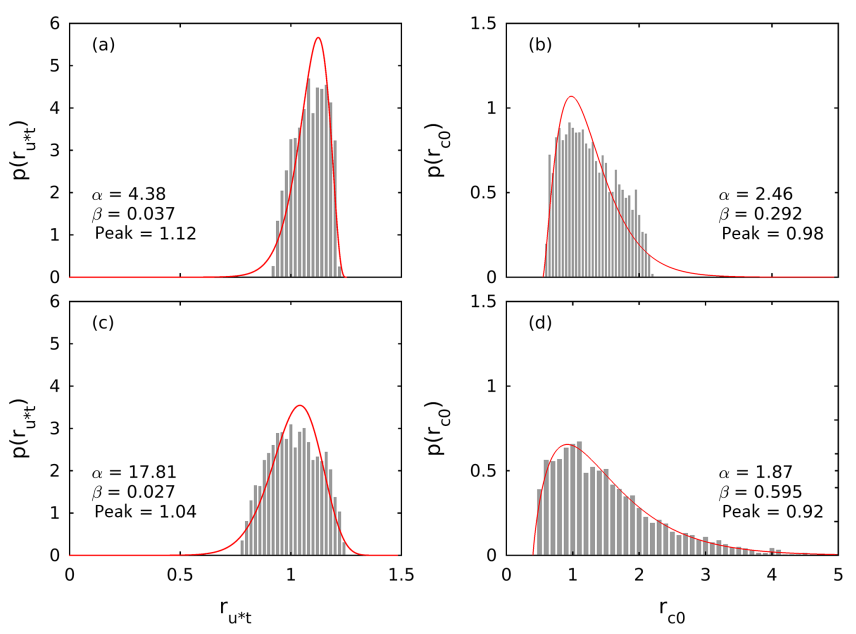

Figure 9. (a) Parameter PDF $p\left(r_{u_{* t}}\right)$ for 1 min averaged saltation fluxes; (b) as (a) but for $p\left(r_{c_{0}}\right)$; (c) and (d), as (a) and (b) but for $30 \mathrm{~min}$ averaged saltation fluxes.

shear stress), the randomness of threshold friction velocity and soil particle size distribution (representing particle response to forcing). Studies have shown, for instance, that small changes in soil moisture can have a large influence on saltation (Ishizuka et al., 2008) and soil moisture in the very top soil layer can vary significantly over relatively short time periods. Over the period of 18 days during JADE, soil moisture in the top $0.05 \mathrm{~m}$ layer varied between 0.02 and $0.04 \mathrm{~m}^{3} \mathrm{~m}^{-3}$ (4 and $8 \%$ in relative soil moisture, assuming a saturation soil moisture of $0.5 \mathrm{~m}^{3} \mathrm{~m}^{-3}$ ). In this study, the influence of soil moisture on saltation is accounted for via Eq. (1) using the soil moisture measurements in the top $0.05 \mathrm{~m}$ layer (see also Fig. 4a in Shao et al., 2011). While measured soil moisture is used in the wind erosion model, the randomness associated with its spatial-temporal variations is not, which is most likely reflected in the stochasticity of $u_{* t}$.

The stochasticity of $c_{0}$ arises because saltation fluctuates depending on turbulence and particle size. To demonstrate this, we divided the time series of the saltation fluxes into two subsets, one with $Q_{D, i} \leq 3 \mathrm{~g} \mathrm{~m}^{-1} \mathrm{~s}^{-1}$ representing weak saltation and one with $Q_{D, i}>3 \mathrm{~g} \mathrm{~m}^{-1} \mathrm{~s}^{-1}$ representing significant saltation. This separation is arbitrary but sufficient for making the point that $c_{0}$ depends on $u_{*}$, which is also a measure of turbulence intensity. The parameter PDFs, $p\left(r_{u_{* t}}\right)$ and $p\left(r_{c_{0}}\right)$, for the subset $Q_{D, i} \leq 3 \mathrm{~g} \mathrm{~m}^{-1} \mathrm{~s}^{-1}$ is shown in Fig. 10. For $Q_{1 \text { min }}$ and $Q_{30 \text { min }}$, the most frequent $r_{u_{* t}}$ values are now 0.99 and 0.85 , somewhat smaller than the estimated values for the full set (Fig. 9). In comparison, the most frequent $r_{c_{0}}$ values are now 0.30 and 0.29 , three to four times smaller than for the case when the full set is considered (Fig. 9). This suggests that $c_{0}$ has a clear dependency on $u_{*}$ and is smaller for smaller $u_{*}$. This is because saltation is more intermittent in the case of smaller $u_{*}$ (i.e. smaller excess shear stress) and thus, $c_{0}$, a descriptor of the relation 

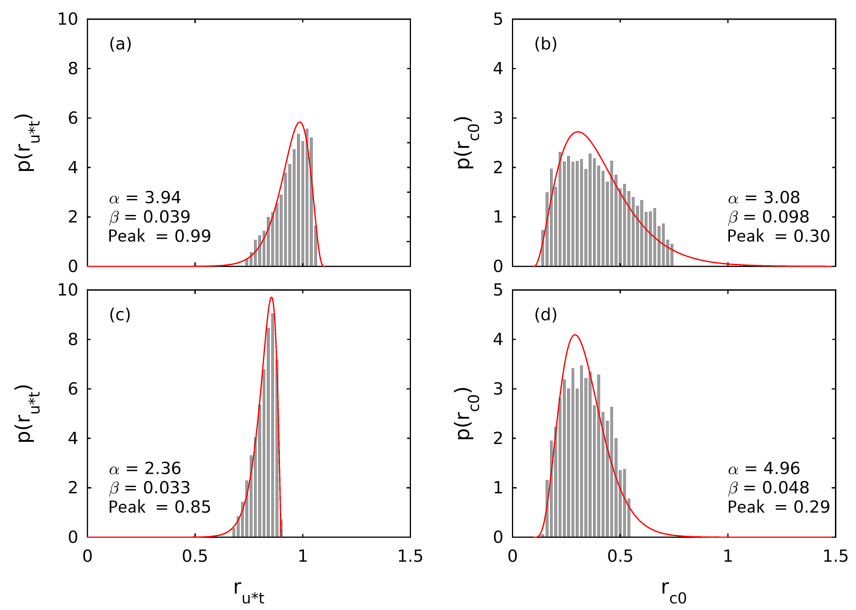

Figure 10. As Fig. 9 but estimated using the time series of saltation fluxes which satisfy $Q_{D, i} \leq 3 \mathrm{~g} \mathrm{~m}^{-1} \mathrm{~s}^{-1}$.

between time-averaged saltation flux and friction velocity, is smaller for more intermittent saltation.

We fit the PDFs, $p\left(r_{u_{* t}}\right)$ and $p\left(r_{c_{0}}\right)$, for individual particle size bins and found that the most frequent $r_{u_{* t}}$ values do not differ substantially among the particle sizes, but $r_{c_{0}}$ depends systematically on particle size. For example, the most frequent $r_{c_{0}}$ values for 101, 151, 203, 315 and $398 \mu \mathrm{m}$ are $0.5,1.3,1.7,3.1$ and 4.0. These values are obtained by first estimating $p\left(r_{c_{0}}\right)$ for the individual particle size bins with the measured saltation flux for the corresponding bins and then normalising $p\left(r_{c_{0}}\right)$ with the mass fraction of the size bins of the parent soil. A least squares curve fitting shows that the most frequent $r_{c_{0}}$ value depends almost perfectly $\left(R^{2}=0.996\right)$ linearly on particle size:

$r_{c_{0}}=0.012 d-0.59$,

for the particle size range (100 to $400 \mu \mathrm{m}$ ) we tested, with $d$ being particle size in $\mu \mathrm{m}$.

We have shown that both $u_{* t}$ and $c_{0}$ satisfy certain PDFs that depend on the properties of the surface, atmospheric turbulence and soil particle size. Figure 9 shows that for a fixed choice of $u_{* t}$ and $c_{0}$, even if they are optimally chosen, a portion of the measurements cannot be represented by the model. Then, how does the saltation model perform if a single fixed $u_{* t}$ and a single fixed $c_{0}$ are used, as is often the case in aeolian models? The $p(Q)$ computed using the model and derived from the JADE measurements are shown for $Q_{1 \mathrm{~min}}$ and $Q_{30 \mathrm{~min}}$ in Fig. 11. The model is applied to estimate the saltation flux for individual particle size groups using the optimally estimated $u_{* t}$ and $c_{0}$ (with $r_{u_{* t}}=1.12$ and $r_{c_{0}}=1.04$ for $Q_{1 \mathrm{~min}}$, and $r_{u_{* t}}=1.04$ and $r_{c_{0}}=0.92$ for $Q_{30 \mathrm{~min}}$ ), and the total saltation flux is computed by integration over all particle size groups, i.e. using Eq. (3). Figure 11 shows that for this option, the model overpredicts the probability of large $Q$ but underpredicts the probability of small

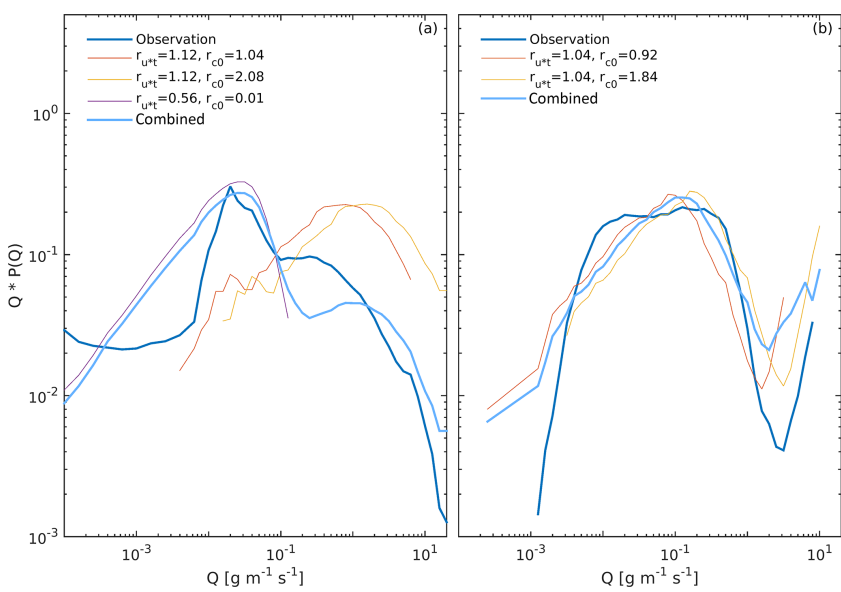

Figure 11. (a) Probability density functions of observed $Q$ and simulated $Q$ for $1 \mathrm{~min}$ averages with several choices of $r_{u_{* t}}$ and $r_{c_{0}}$; (b) as (a) but for $30 \mathrm{~min}$ averages.

$Q$ in both cases of $Q_{1 \mathrm{~min}}$ and $Q_{30 \mathrm{~min}}$. Obviously, to better reproduce the $Q_{1 \mathrm{~min}}$ and $Q_{30 \mathrm{~min}}$ PDFs, more values of $r_{u_{* t}}$ and $r_{c_{0}}$ sampled from the parameter PDFs are required. We have therefore modelled $Q_{1}$ min with other choices of $r_{u_{* t}}$ (1.12 and 0.56) and $r_{c_{0}}(2.08,0.01)$ and plotted the corresponding $Q_{1 \mathrm{~min}}$ PDFs as well as the averaged $Q_{1 \mathrm{~min}}$ PDF of the three simulations. Similarly, we performed $Q_{30 \text { min }}$ model simulations with other $r_{u_{* t}}$ (1.04) and $r_{c_{0}}$ (1.84) values and examined the $Q_{30 \mathrm{~min}}$ PDFs. With the additional choices of the $r_{u_{* t}}$ and $r_{c_{0}}$ values, the $Q_{1 \min }$ and $Q_{30 \min }$ PDFs can be better reproduced.

\section{Summary}

In this paper, we used the JADE data on saltation fluxes (resolution $1 \mathrm{~s}$ ) and frictional velocity (resolution $1 \mathrm{~min}$ ) to analyse the statistical behaviour of turbulent saltation and estimate the probability distribution of two important parameters in a saltation model, namely the threshold friction velocity, $u_{* t}$, and saltation coefficient, $c_{0}$.

Saltation fluxes show rich variations on different scales. It is found that while the widely used $Q \sim u_{*}^{3}$ relationship holds in general, it can vary significantly between different wind erosion events. In several wind erosion events observed in JADE, saltation hysteresis occurred. We examined the probability density function of the saltation fluxes, $p(Q)$, and found that it generally behaves like $Q^{-\alpha}$ with $\alpha \sim 1$. For $Q_{1 \mathrm{~s}}$, there is a distinct change in $\alpha$ at $Q=3 \sim 4 \mathrm{~g} \mathrm{~m}^{-1} \mathrm{~s}^{-1}$ with $\alpha \sim 1$ for smaller $Q$ and $\alpha \sim 4.0$ larger $Q$. It is shown that $p(Q)$ is dependent on the averaging time intervals as a consequence of saltation intermittency.

We introduced the saltation intermittency functions $\gamma_{a}\left(u_{* t}\right), \gamma_{b}\left(u_{*}\right)$ and redefined saltation intermittency $\gamma_{c}$ as the fraction of time during which saltation occurs at a given point in a given time period, and computed these saltation 
intermittency measures using the JADE saltation flux measurements. It is found that $\gamma_{a}\left(u_{* t}\right)$ is one at $u_{* t}=0$ and decreases to zero at about $u_{* t}=0.5 \mathrm{~m} \mathrm{~s}^{-1}$. For $u_{* t}=0.2 \mathrm{~m} \mathrm{~s}^{-1}$, $\gamma_{a}$ is 0.35 . For $Q_{1 \mathrm{~min}}, \gamma_{b}\left(u_{*}\right)$ increases from about 0.6 at $u_{*} \sim 0.1 \mathrm{~m} \mathrm{~s}^{-1}$ to about one at $u_{*}=0.3 \mathrm{~m} \mathrm{~s}^{-1}$. This shows that a considerable fraction of the saltation fluxes occurs at small friction velocity and saltation is more intermittent under weak wind conditions and is almost non-intermittent for $u_{*}>0.3 \mathrm{~m} \mathrm{~s}^{-1}$. It is found that $\gamma_{b}\left(u_{*}\right)$ increased with decreasing $u_{*}$ for $u_{*}<0.1 \mathrm{~m} \mathrm{~s}^{-1}$, which is unexpected. Overall, $\gamma_{c}$ is found to be around 0.73 . We computed $\gamma_{c}$ as a function of particle size and found that $\gamma_{c}(d)$ decreases with $d$; i.e. the saltation of larger particles is more intermittent. Also, $\gamma_{c}(d)$ increases with increased averaging time intervals, implying that the small-scale features of turbulence play an important role in intermittent saltation.

The power spectra of $Q$ and $u_{*}$ are found to have qualitatively similar behaviour. Both have a maximum at about $10^{-5} \mathrm{~Hz}$, a minimum at about $10^{-4} \mathrm{~Hz}$ and another peak at about $2 \times 10^{-3} \mathrm{~Hz}$. The maximum at $10^{-5} \mathrm{~Hz}$ is related to the diurnal to synoptic events that drive wind erosion episodes, the minimum at $10^{-4} \mathrm{~Hz}$ is due to the lack of turbulent wind fluctuations at the timescale of several hours, while the peak at $2 \times 10^{-3} \mathrm{~Hz}$ is caused by minute-scale gusts/large eddies in turbulent flows. The power of the saltation rapidly decreases with frequency and becomes relatively weak at frequencies of $0.1 \mathrm{~Hz}$.

The posterior PDFs of the two parameters were estimated using the DREAM algorithm applied to the JADE saltation flux measurements. While both $u_{* t}$ and $c_{0}$ have clear physical interpretations, they are both stochastic parameters that satisfy certain parameter PDFs. They also depend on the intervals of time averaging. Both $u_{* t}$ and $c_{0}$ for $Q_{1 \text { min }}$ are larger than for $Q_{30 \mathrm{~min}}$. The PDF of $u_{* t}$ shows that it has a most frequent value close to the theoretical value but can vary over a range of 20 to $30 \%$. The PDF of $c_{0}$ shows scatter over a wide range and it is unlikely that a universal $c_{0}$ exists. In a saltation model, even if the optimally estimated $c_{0}$ is used, considerable scatter between the model and the data would remain. The likely reason for the stochasticity in $u_{* t}$ may be the temporal and spatial variations of particle cohesion, surface roughness, particle shape, etc., which cannot be well represented by a fixed deterministic value, and the relatively large uncertainty in $c_{0}$ may be because this parameter depends on additional factors (e.g. $u_{*}$ and soil particle size distribution) and is related to the fluctuations and intermittency of saltation. It may also be that saltation in reality is never in equilibrium as Bagnold (1941), Kawamura (1964) and Owen (1964) conceptualised, because due to turbulence, sand grains are continuously entrained at different rates into the airflow, and a continuous flow- and particle-motion feedback takes place. As a consequence, it is difficult to treat $c_{0}$ as a universal constant.

In this study, we highlighted the need to better understand saltation as a turbulent process and the stochasticity of saltation model parameters. The concept of threshold friction velocity as a stochastic variable was put forward in Shao (2001). Raffaele et al. (2016) examined the PDF of $u_{* t}$ using data compiled from publications. Raffaele et al. (2018) studied how $u_{* t}$ uncertainties propagate in saltation flux calculations and reported that in the case of small excess shear stress, all models they tested amplify the uncertainty in estimated saltation flux, especially for coarse sand. This finding is consistent with our notion that $c_{0}$ is also a stochastic variable. Due to the stochasticity of the model parameters, the saltation model cannot reproduce the observation even with the optimally estimated parameters (e.g. underestimation of weak saltation fluxes and over estimation of strong saltation fluxes). A combination of several pairs of model parameters appears to be required to reasonably reproduce the PDFs of saltation fluxes.

Our estimates of the parameter uncertainties are based on the data of a relatively simple aeolian surface. For more complex surfaces, we expect the parameter uncertainties to be even more pronounced.

Data availability. The JADE data used in this study are available at the public data repository https://doi.org/10.17632/896tx3ns6x.1 (Ishizuka, 2018).

Competing interests. The authors declare that they have no conflict of interest.

Acknowledgements. This research is funded by the National Natural Science Foundation of China (no. 41571090, 41201539). The data used in this study were obtained from JADE (the Japan Australian Dust Experiment) by Masahide Ishizuka, Masao Mikami, John F. Leys, Yutaka Yamada, and Stephan Heidenreich. We are grateful to Philipp Schlüter and Qian Xia for support with data processing. We also wish to thank John Gillies, Martina Klose and an anomalous referee for their helpful comments which prompt us to rework a number of issues presented in the first version of the paper.

Edited by: Bernhard Vogel

Reviewed by: John Gillies and one anonymous referee

\section{References}

Anderson, R. S. and Haff, P. K.: Simulation of Eolian Saltation, Science, 241, 820-823, https://doi.org/10.1126/science.241.4867.820, 1988.

Bagnold, R. A.: The Physics of Blown Sand and Desert Dunes, Methuen, London, 265 pp., 1941

Butterfield, G. R.: Grain transport rates in steady and unsteady turbulent airflows, Acta Mech., Suppl. 1, 97-122 1991.

Davidson-Arnott, R. G. D. and Bauer, B. O.: Aeolian sediment transport on a beach: Thresholds, intermittency, and high frequency variability, Geomorphology, 105, 117-126, 2009. 
Dupont, S., Bergametti, G., Marticorena, B., and Simoëns, S.: Modeling saltation intermittency, J. Geophys. Res.-Atmos., 118, 7109-7128, https://doi.org/10.1002/jgrd.50528, 2013.

Ellis, J. T., Sherman, D., Farrell, E. J., and Li, B. L.: Temporal and spatial variability of aeolian sand transport: Implications for field measurements, Aeolian Res., 3, 379-387, https://doi.org/10.1016/j.aeolia.2011.06.001, 2012.

Fécan, F., Marticorena, B., and Bergametti, G.: Parametrization of the increase of the aeolian erosion threshold wind friction velocity due to soil moisture for arid and semi-arid areas, Ann. Geophys., 17, 149-157, https://doi.org/10.1007/s00585-999-0149-7, 1999.

Gillette, D. A., Hardebeck, E., and Parker, J.: Large-scale variability of wind erosion mass flux rates at Owens Lake 2. Role of roughness change, particle limitation, change of threshold friction velocity, and the Owen effect, J. Geophys. Res., 102, 25989-25998, 1997.

Ishizuka, M.: “JADE data”, Mendeley Data, v1, https://doi.org/10.17632/896tx3ns6x.1, 2018.

Ishizuka, M., Mikami, M., Leys, J. F., Yamada, Y., Heidenreich, S., Shao, Y., and McTainsh, G. H.: Effects of soil moisture and dried raindroplet crust on saltation and dust emission, J. Geophys. Res., 113, D24212, https://doi.org/10.1029/2008JD009955, 2008.

Ishizuka, M., Mikami, M., Leys, J. F., Shao, Y., Yamada, Y., and Heidenreich, S.: Power law relation between size-resolved vertical dust flux and friction velocity measured in a fallow wheat field, Aeolian Res., 12, 87-99, https://doi.org/10.1016/j.aeolia.2013.11.002, 2014.

Kawamura, R.: Study of sand movement by wind, in: Hydraulic Eng. Lab. Tech. Rep., University of California, Berkeley, HEL2-8, 99-108, 1964.

Klose, M., Shao, Y., Li, X., Zhang, H., Ishizuka, M., Mikami, M., and Leys, J. F.: Further development of a parameterization for convective turbulent dust emission and evaluation based on field observations, J. Geophys. Res.-Atmos., 119, 10441-10457, https://doi.org/10.1002/2014JD021688, 2014.

Kok, J. F., Mahowald, N. M., Fratini, G., Gillies, J. A., Ishizuka, M., Leys, J. F., Mikami, M., Park, M.-S., Park, S.-U., Van Pelt, R. S., and Zobeck, T. M.: An improved dust emission model - Part 1: Model description and comparison against measurements, Atmos. Chem. Phys., 14, 13023-13041, https://doi.org/10.5194/acp-14-13023-2014, 2014.

Leys, J. F.: Wind erosion processes and sediments in southeastern Australia, PhD Thesis, Griffith University, Brisbane, 1998.

McKenna-Neuman, C., Lancaster, N., and Nickling, W. G.: The effect of unsteady winds on sediment transport on the stoss slope of a transverse dune, Silver Peak, NV, USA, Sedimentology, 47, 211-226, 2000.

McNaughton, K. G. and Laubach, J.: Power Spectra and Cospectra for Wind and For Wind and Scalars in a Disturbed Surface Layer at the Base of an Advective Inversion, Bound.-Lay. Meteorol., 96, 143-185, 2000.

Namikas, S. L., Bauer, B. O., and Sherman, D.: Influence of averaging on shear velocity estimates for aeolian transport modeling, Geomorphology, 53, 235-246, https://doi.org/10.1016/S0169555X(02)00314-8, 2003.
Owen, R. P.: Saltation of uniform grains in air, J. Fluid. Mech., 20, 225-242, 1964.

Raffaele, L., Bruno, L., Pellerey, F., and Preziosi, L.: Windblown sand saltation: A statistical approach to fluid threshold shear velocity, Aeolian Res., 23, 79-91, https://doi.org/10.1016/j.aeolia.2016.10.002, 2016.

Raffaele, L., Bruno, L., and Wiggs, G. F. S.: Uncertainty propagation in aeolian processes: From threshold shear velocity to sand transport rate, Geomorphology, 301, 28-38, https://doi.org/10.1016/j.geomorph.2017.10.028, 2018.

Raupach, M. R., Gillette, D. A., and Leys, J. F.: The effect of roughness elements on wind erosion thresholds, J. Geophys. Res., 98, 3023-3029, 1993.

Sadegh, M. and Vrugt, J. A.: Approximate Bayesian computation using Markov Chain Monte Carlo simulation: DEARM $(\mathrm{ABC})$, Water Resour. Res., 50, https://doi.org/10.1002/2014WR015386, 2014.

Shao, Y.: Physics and Modelling of Wind Erosion, 1st Edn., Kluwer Academic Publishers, Dordrecht, 2001.

Shao, Y. and Lu, H.: A simple expression for wind erosion threshold friction velocity, J. Geophys. Res., 105, 22437-22443, 2000.

Shao, Y. and Mikami, M.: Heterogeneous Saltation: Theory, Observation and Comparison, Bound.-Lay. Meteorol., 115, 359-379, https://doi.org/10.1007/s10546-004-7089-2, 2005.

Shao, Y., Ishizuka, M., Mikami, M., and Leys, J. F.: Parameterization of size-resolved dust emission and validation with measurements, J. Geophys. Res., 116, D08203, https://doi.org/10.1029/2010JD014527, 2011.

Sherman, D., Li, B. L., Ellis, J. T., and Swann, C.: Intermittent aeolian saltation: A protocol for quantification, Geogr. Rev., 108, 296-314, https://doi.org/10.1111/gere.12249, 2017.

Storn, R. and Price, K.: Differential Evolution - a simple and efficient heuristic for global optimization over continuous spaces, J. Global Optim., 11, 341-359, 1997.

Stout, J. E. and Zobeck, T. M.: Intermittent saltation, Sedimentology, 44, 959-970, 1997.

Stull, R. B.: An Introduction to Boundary Layer Meteorology, Kluwer Academic Publishers, Dordrecht, 1988.

Vrugt, J. A. and Sadegh, M.: Toward diagnostic model calibration and evaluation: Approximate Bayesian computation, Water Resour. Res., 49, 4335-4345, https://doi.org/10.1002/wrcr.20354, 2013.

Vrugt, J. A., ter Braak, C. J. F., Diks, G. H., Robinson, B. A., and Hyman, J. M.: Accelerating Markov Chain Monte Carlo Simulation by Differential Evolution with Self-Adaptive Randomized Subspace Sampling, Int. J. Nonlinear. Sci., 10, 273-290, https://doi.org/10.1515/IJNSNS.2009.10.3.273, 2011.

White, B. R.: Soil transport by winds on Mars, J. Geophys. Res., 84, 4643-4651, 1979.

Yamada, Y., Mikami, M., and Nagashima, H.: Dust particle measuring system for streamwise dust flux, J. Arid Land Studies, 11, 229-234, 2002. 